\title{
Qualidade da informação contábil e sustentabilidade nas companhias brasileiras listadas na BM\&FBovespa
}

\section{Quality of Accounting Information and Sustainability in Brazilian Companies listed on the BM\&FBovespa}

\author{
Gildo Rodrigues da Silva ${ }^{1}$ \\ Fábio Moraes da Costa²
}

\section{Resumo}

O objetivo deste artigo foi avaliar se a entrada no Índice de Sustentabilidade Ambiental (ISE) leva à redução no gerenciamento de resultados ou se esta seria apenas uma forma indireta de reunir fatores já ligados diretamente com a qualidade da informação contábil. O estudo analisou se as empresas consideradas sustentáveis e participantes no ISE possuem menor nível de gerenciamento de resultados (GR). Como proxy de sustentabilidade, foi utilizado o ISE, e, como proxy de qualidade, foi utilizado o GR. Foram realizadas 797 observações com dados coletados na BM\&FBovespa pela ferramenta da Economática no período pós IFRS, de 2010 a 2012. No artigo, foi utilizado o modelo Jones (1991), modificado por Dechow, Sloan e Sweeny (1995), e a Teoria da Divulgação. Os resultados sugerem uma relação negativa entre os accruals discricionários e a participação no ISE, indicando que não é a entrada no ISE que leva as empresas a gerenciarem menos os resultados, mas sim os critérios estabelecidos pela BM\&FBovespa para participação no índice, tais como relatório de sustentabilidade, tamanho e nível de governança. Assim, a participação no ISE poderia ser utilizada pelo mercado como uma métrica de sinalização para capturar a qualidade da informação, possibilitando ganho em termos de resumo e redução dos custos de transação. Os resultados corroboram com as pesquisas nacionais e internacionais ao adicionar a variável sustentabilidade como uma das proxies de qualidade da informação contábil, assim como as variáveis tamanho da empresa e atributos de governança corporativa.

Mestre em Ciências Contábeis - Fucape Business School. Afiliação: Fucape Business School. Vitória/ES. Brasil. Lattes: http://lattes.cnpq.br/436624357650941 E-mail: gilldorodrigues@gmail. com

2 Doutor em Ciências Contábeis - Fucape Business School. Afiliação: Fucape Business School. Vitória/ES. Brasil. Lattes: http://lattes.cnpq.br/6836059339343875 E-mail: fabio@fucape.br 
Palavras-chave: Índice de Sustentabilidade Ambiental. Qualidade da informação contábil. Gerenciamento de resultados.

\section{Abstract}

The aim of this paper was to assess whether the entry in the Environmental Sustainability Index (ISE) leads to a reduction in earnings management, or whether this was only an indirect way of gathering already factors directly linked to the quality of accounting information. The study examined whether sustainable companies and participants in the ISE have lower level of earnings management (GR). As proxy sustainability ISE was used, and as a quality proxy we used GR. 797 were carried out observations with data collected on the BM\&FBovespa by Economática tool post IFRS period 2010 to 2012. In the article we used the Jones (1991) model modified by Dechow, Sloan and Sweeny (1995) and the Theory of Communication. The results suggest a negative relationship between discretionary accruals and participation in ISE, indicating that it is not entering the ISE leading companies manage less results, but the criteria established by BM\&FBovespa to participate in the index, such as report sustainability, size and level of governance. Thus, participation in the ISE could be used by the market as a signal metric to capture the quality of information, allowing gain in summary and reduction of transaction costs. The results corroborate national and international research, adding the variable sustainability as one of the quality of accounting information proxies, as well as the size of the company variables and attributes of corporate governance.

Keywords: Environmental Sustainability Index. Quality of accounting information. Earnings management.

\section{Introdução}

A informação contábil é relevante para o mercado financeiro, e a qualidade da divulgação influencia a qualidade das decisões de investimento. Por outro lado, dado que a gestão tem poder discricionário, suas escolhas acabam por afetar a qualidade dos eventos representados por meio de informações contábeis (DECHOW; GE; SCHRAND, 2010; BOUBAKER, 2011; WARFIELD; WILD, 1995).

O Índice de Sustentabilidade Ambiental (ISE) é um indicador utilizado pelo mercado para analisar comparativamente o desempenho das empresas da BM\&FBovespa, objetivando auxiliar os investidores e 
outros grupos de interesse na diferenciação do desempenho de uma empresa sustentável em relação àquelas consideradas não sustentáveis.

Vários trabalhos comparam empresas sustentáveis com as classificadas como não sustentáveis, com foco em seu desempenho, rentabilidade, risco e retorno (por exemplo, FREGONESI; NOVELINI, 2013; NOBRE; RIBEIRO, 2013; NOVA; NUNES, 2012; MANHÃES; MELO, 2012; BRESSAN; COLARES; LAMOUNIER, 2012; FUNCHAL; NOSSA; TEIXEIRA, 2011; NUNES; PORTELA; REZENDE, 2008). Outros artigos comparam a qualidade da informação da contabilidade, evidenciando o efeito da qualidade sobre a eficiência, redução do investimento, riscos à continuidade dos negócios, omissão de indicadores, assimetria da informação contábil, quantidade de informações ambientais e desempenho (por exemplo, BACHMANN; CARNEIRO; ESPEJO, 2013; MAZZA; MOTA; OLIVEIRA, 2013; CALVANCANTE; CALVANCANTE; PAULO, 2013; FREITAS; LIMA; SANTOS, 2011; DECHOW; GE; SCHRAND, 2010; ANTUNES; COSTA; NOSSA; TEIXEIRA, 2010; PAULO, 2007; DECHOW; SLOAN; SWEENY, 1996).

Todavia, as pesquisas anteriores não decorreram sobre a qualidade da informação contábil (QIC) e a sustentabilidade empresarial tendo como métrica gerenciamento de resultados (GR) e o ISE da BM\&FBovespa, nem as possíveis implicações dos resultados dessa associação.

Embora essas pesquisas encontrassem diferenças significantes entre as empresas consideradas sustentáveis e as não consideradas, esses resultados ainda representam um mercado insipiente e imaturo, pois a BM\&FBovespa não reage na mesma proporção dos outros mercados internacionais no que tange à divulgação de informações (BRESSAN; COLARES; LAMOUNIER, 2012).

Segundo Dechow, Ge e Schrand (2010) e Byard e Weintrop (2006), várias proxies estão associadas à qualidade da informação contábil, como tamanho da empresa e atributos de governança corporativa, dentre outras. Porém, essas mesmas proxies também fazem parte do questionário respondido pelas empresas consideradas sustentáveis. 
Nesse contexto, apresenta-se o seguinte questionamento: as empresas que estão incluídas no ISE possuem melhor qualidade da informação contábil em relação às demais empresas da BM\&FBovespa?

O objetivo desta pesquisa é analisar se as empresas inclusas no ISE possuem menor nível de gerenciamento de resultados e, portanto, maior qualidade da informação contábil em relação às demais empresas da BM\&FBovespa.

Para diferenciar as práticas de sustentabilidade das demais empresas, utilizou-se o ISE como parâmetro, pois a BM\&FBovespa divulga esse índice como referência de melhores práticas de sustentabilidade. Já como proxy de QIC, foi estimado o nível de GR.

A busca por informações de qualidade não pode ser entendida como a busca por quantidade, sendo interessante obter informações relevantes, pois a deficiência ou excesso de informações inúteis acarretará prejuízos aos investidores e/ou partes interessadas.

Nascimento e Reginato (2008) elencam os interessados na informação contábil, como: os gestores, investidores, stakeholders, governo, clientes, dentre outros. Contudo, os usuários externos possuem uma desvantagem informacional, tendo em vista a natural distância em que se encontram do ambiente gerador das informações contábeis (FREITAS; LIMA; RIBEIRO; SANTOS;TRETER, 2011; COSTA; SILVA; 2015).

As evidências deste estudo indicam que as empresas inclusas no ISE apresentam menor gerenciamento de resultados. Todavia, existem critérios para uma empresa entrar no ISE, tais como: divulgação de relatório de sustentabilidade, características da sua estrutura de governança, e tamanho. Assim, haveria relação negativa entre accruals e ISE em função de estarem estes critérios também ligados ao GR e, consequentemente, maior QIC.

O ISE, portanto, poderia ser utilizado como uma proxy para sinalização de qualidade, o que por sua vez, poderia trazer ganhos ao mercado em termos de resumo e redução dos custos de transação. 
Demonstrando assim, que os critérios utilizados são determinantes da qualidade da informação contábil e não o ISE.

Neste artigo, os achados corroboram com as pesquisas de Dechow, Ge e Schrand (2010) e Byard e Weintrop (2006), ao adicionar a variável sustentabilidade como proxie de qualidade da informação contábil, assim como as variáveis tamanho da empresa e atributos de governança corporativa.

A Teoria da Sinalização de Spence (1973) foi empregada para a interpretação dos resultados do trabalho, pois as empresas criam formas de emitirem sinais aos investidores e ou partes interessadas.

A pesquisa é relevante, pois as empresas usam a qualidade da informação contábil como meio de diminuir a assimetria de informações entre as partes envolvidas, melhorando, por conseguinte, os possíveis conflitos de interesse. Além disso, o mercado financeiro visualiza o relatório de sustentabilidade como uma forma de transparência de negócio, demonstrando que empresas envolvidas com a sustentabilidade possuem facilidade para se diferenciarem das demais empresas em termos de resumo, riscos menores e simplificação das informações sobre os investimentos, gerando, assim, maior confiança para os stakeholders (LEON; MOON, 2007; GARCIA; ORSATO, 2013).

\section{Referencial teórico}

\subsection{Sustentabilidade Empresarial}

O Índice de Sustentabilidade Empresarial (ISE), criado em 2005, é composto por até 40 empresas dentre as emissoras das 200 ações mais líquidas na BM\&FBovespa. A participação no indicador não é obrigatória, mas voluntária, indicando as empresas que estão comprometidas com a sustentabilidade (BACARJI; MARCONDES, 2010; FUNCHAL; NOSSA; TEIXEIRA, 2011; BM\&FBOVESPA, 2013).

O ISE possui o objetivo de aferir o retorno de ações das empresas comprometidas com as práticas de sustentabilidade que compõem 
a carteira, ponderadas pela liquidez (BACARJI; MARCONDES, 2010; MACEDO; MACHADO; MACHADO, 2012 e 2013; BM\&FBOVESPA, 2013).

A seleção das empresas para o ISE segue o triple bottom line desenvolvido por Elkington(2004) e o questionário elaborado pela consultoria inglesa SustainAbility, o qual faz menção aos elementos avaliativos ambientais, sociais e financeiros, bem como publicações de cunho social e governança corporativa, com seus atos normatizados pela Fundação Getúlio Vargas (BACARJI; MARCONDES, 2010; BM\&FBOVESPA, 2013). As respostas das empresas ao questionário são analisadas estatisticamente pela "análise de clusters" que, após a identificação das companhias com melhor desempenho, indicam as 40 (quarenta) empresas que farão parte da carteira ISE (NUNES; PORTELA; REZENDE, 2008; BM\&FBOVESPA, 2013; SILVA; COSTA, 2015).

Uma empresa deve, segundo a BM\&FBovespa, atender aos seguintes critérios para ser incluída no ISE (BM\&FBOVESPA, 2013):

a) ser uma das 200 ações com maior índice de negociabilidade apurados nos doze meses anteriores ao início do processo de reavaliação;

b) ter sido negociada em pelo menos $50 \%$ dos pregões ocorridos nos doze meses anteriores ao início do processo de reavaliação;

c) atender aos critérios de sustentabilidade referendados pelo Conselho do ISE.

O ISE engloba os relacionamentos das empresas com: empregados, comunidades, fornecedores, governança corporativa e o impacto a atividades empresariais, tendo em vista que oferece aos investidores uma possível sinalização das ações empresariais desenvolvidas em prol da sustentabilidade empresarial (ENSSLIN; GALLON,2008; BM\&FBOVESPA, 2013; NOBRE; RIBEIRO, 2013).

Nova e Nunes (2012) investigaram a possível ocorrência de diferenças significativas nos índices de companhias sustentáveis em face 
de companhias não sustentáveis usando o ISE. Os resultados indicaram a inexistência de diferenças dos indicadores contábeis relacionados com as empresas consideradas sustentáveis em detrimento daquelas não consideradas sustentáveis.

A pesquisa de Teixeira e Nossa (2010) sugerem uma redução do custo de capital das empresas que se envolvem com a sustentabilidade, aumentando, por conseguinte, a credibilidade e possibilitando a redução ou até mesmo a eliminação de passivos ambientais.

Novelini e Gregonessi (2013) analisaram a divulgação de informação de investimentos sustentáveis das empresas. Os resultados sugerem que as informações mais divulgadas são as que possuem, em seu bojo, um impacto positivo para as empresas (COSTA; SILVA, 2015).

A pesquisa de Macedo, Machado e Machado (2012) sugere uma relação positiva entre os investimentos sustentáveis e o ingresso das empresas no ISE, sugerindo, inclusive, que empresas que investem seus recursos em função da comunidade, seja espontaneamente, seja meio dos tributos, possuem maiores chances de participarem do ISE.

Nunes, Portela e Rezende (2008), analisando a rentabilidade dos índices do mercado brasileiro em detrimento do ISE sob a ótica da teoria dos stakeholders, constataram que as companhias que apresentam maiores práticas sustentáveis teriam índices com retornos semelhantes aos das demais carteiras (MANHÃES; MELO, 2012).

Os achados de Andrade, Bressan e Iquiapaza (2013) evidenciam que empresas que possuem maior tamanho, rentabilidade e fazem parte dos setores de maior impacto ambiental, possuem maiores chances de aderirem ao ISE.

\subsection{Qualidade da Informação Contábil (QIC)}

Mazza, Mota e Oliveira (2013) sugerem que as empresas brasileiras encontram-se numa fase inicial de desenvolvimento sustentável, apontando a omissão de dados e a falta de análise crítica dos resultados como propulsora da baixa qualidade das informações divulgadas. Os 
resultados ainda evidenciam a existência de camuflagem de dados, por meio de omissão de indicadores e de resultados tidos como negativos transformados em ações motivadoras pelas companhias (COSTA; SILVA, 2015).

A informação contábil é considerada relevante para as empresas que estão sujeitas a um volume considerado de ameaças, que podem fazer com que estas incorram em risco à continuidade dos seus negócios, ou podem provocar danos que comprometeriam a competitividade, rentabilidade e até mesmo o cumprimento regular das leis (ADACHI; BIAGI;SANTOS; UREL; VENDRAME, 2009).

Biddle e Hilary (2006) constataram que maior qualidade de contabilidade melhora a eficiência do investimento, porque é associado à menor sensibilidade do fluxo de investimento de dinheiro, e que, por isso, seu efeito seria mais forte nas economias que dependem mais do financiamento de capital público (EUA) do que nos países em que os bancos e os credores são os principais fornecedores de capital (Japão).

Para Szüster e Szüster (2005), os demonstrativos da contabilidade devem evidenciar e apresentar às partes interessadas informações de qualidade e credibilidade que, ao mesmo tempo, ampliem a capacidade informativa dos investidores e mantenha-se fidedigna à realidade praticada no dia a dia das empresas (COSTA; SILVA, 2015).

Segundo Ballesta, Solano e Teruel (2009), a qualidade da informação da contabilidade pode contribuir para a redução dos efeitos negativos da assimetria de informação e também dos custos de seleção adversa, permitindo, em tese, que as empresas reduzam o nível de reservas de caixa.

Paulo, Cavalcanti e Paulo (2013) evidenciaram uma relação positiva entre qualidade da informação e dados contábeis conservadores; por outro lado, no gerenciamento, quanto mais alta a qualidade da informação, menor seria a discricionariedade.

Para Antunes e Costa (2007), os dados analisados sugerem que as empresas que fazem parte dos níveis diferenciados de Governança 
da Bovespa (NDGB) não apresentaram melhores métricas de QIC quando comparadas com as empresas não inclusas nos demais NDGB.

Santos, Dani e Filho (2011) analisaram as empresas listadas no segmento de Tecnologia da Informação (TI) da BM\&FBovespa no ano de 2010. Os resultados da pesquisa sugerem não haver melhor desempenho nas empresas analisadas no que tange o nível da QIC.

Pereira, Carvalho e Parente (2011) investigaram a preocupação das empresas em evidenciar as informações sustentáveis e constataram um desempenho econômico melhor das empresas que evidenciam as informações socioambientais.

Para Nascimento e Reginato (2008), as informações inseridas nos relatórios de sustentabilidade visam atender às necessidades e expectativas dos stakeholders ou outros usuários dessas informações, ou seja, a informação terá maior utilidade se chegar ao usuário no momento certo e no formato esperado pelo mercado.

\section{Metodologia}

Esta pesquisa classifica-se como um estudo de natureza descritiva, quantitativa e explicativa. Foi analisado se as empresas inclusas no ISE (Índice de Sustentabilidade Empresarial) possuem menor nível de GR e, portanto, maior QIC em relação às demais empresas da BM\&FBovespa (COSTA; SILVA, 2015).

A natureza descritiva procura descrever um objeto ou fenômeno que se encontra em estudo, no caso, todas as companhias abertas listadas na BM\&FBovespa. A característica quantitativa e explicativa ocorre em função de se buscar analisar as relações e associações existentes entre variáveis econômico-financeiras mediante a teoria da sinalização e dos fundamentos estatísticos e matemáticos.

Assim, para o desenvolvimento das regressões propostas no trabalho, e para estimar e calcular os accruals discricionários, utilizou- 
se o modelo proposto por Jones (1991) modificado por Dechow, Sloan e Sweeny (1995), demonstrado na pesquisa de Paulo, Corrar e Martins (2007).

$$
A D C R_{i t}=\alpha+\beta_{1}\left(\Delta R_{i t}-\Delta C R_{i t}\right)+\beta_{2} A_{i t}+\varepsilon_{i t}
$$

$\mathrm{ADCR}_{\text {it }}=$ accruals total da empresa $\mathrm{i}$ no ano $\mathrm{t}$, = receita bruta menos impostos sobre vendas da empresa i no períodot, = Variação da conta clientes (duplicata a receber) da empresa i no períodot, $\mathrm{Al}_{\mathrm{it}}=$ saldo da conta do ativo diferido e imobilizado da empresa i no período $\mathrm{t}, \varepsilon_{\mathrm{it}}=$ erro.

O estudo levou em consideração os anos de 2010 a 2012, período em que as IFRS já haviam sido implementadas no país, os dados das empresas pertencentes ou não pertencentes à carteira do ISE, e as variáveis independentes foram obtidas na BM\&FBovespa utilizando-se o banco de dados da Economática.

Para o desenvolvimento da pesquisa foram realizadas 797 observações de todas as empresas com ações na BM\&FBovespa, e também foram analisados os relatórios de sustentabilidade e o questionário de adesão das empresas ao ISE.

Seguindo os estudos anteriores, como os de Byard e Weintrop (2006), Nunes, Teixeira e Nossa (2009), Dechow, Ge e Schrand (2010) e Gomes (2012), algumas variáveis de controle foram incluídas, como tamanho, endividamento e alto impacto ambiental, que serviram para isolar os efeitos que poderiam atingir as variáveis independentes (COSTA; SILVA, 2015).

Os dados acima coletados foram transcritos para uma tabela do Excel de forma binária, como demonstrado a seguir no quadro 1. 
Quadro 1 - Descrição das variáveis utilizadas no modelo.

\begin{tabular}{|c|c|c|c|}
\hline Variável & Denominação & Construtos & Proxy \\
\hline $\begin{array}{c}\text { ISE } \\
\text { (dependente) }\end{array}$ & Adesão do ISE & $\begin{array}{c}\text { Empresas que } \\
\text { participam do ISE }\end{array}$ & $\begin{array}{c}\text { Variável dummy } \\
D=1 \text { - empresas } \\
\text { participam do ISE } \\
D=0 \text { - empresas não } \\
\text { participam ISE }\end{array}$ \\
\hline $\begin{array}{c}\text { Novo Mercado } \\
\text { (NM) } \\
\text { (Independente) }\end{array}$ & Adesão ao NM & $\begin{array}{c}\text { Empresas que } \\
\text { participam do NM }\end{array}$ & $\begin{array}{c}\text { Variável dummy } \\
D=1-\text { empresas } \\
\text { participam NM } \\
D=0 \text { - empresas não } \\
\text { participam do NM }\end{array}$ \\
\hline $\begin{array}{l}\text { Nível } 1 \text { Bovespa } \\
\text { (Independente) }\end{array}$ & Adesão ao NG1 & $\begin{array}{c}\text { Empresas que } \\
\text { participam do } \\
\text { NG1 }\end{array}$ & $\begin{array}{c}\text { Variável dummy } \\
\text { D=1 - empresas } \\
\text { participam NG1 } \\
\text { D=0 - empresas não } \\
\text { participam do NG1 }\end{array}$ \\
\hline $\begin{array}{l}\text { Nível } 2 \text { Bovespa } \\
\text { (Independente) }\end{array}$ & Adesão ao NG2 & $\begin{array}{c}\text { Empresas que } \\
\text { participam do } \\
\text { NG2 }\end{array}$ & $\begin{array}{c}\text { Variável dummy } \\
D=1 \text { - empresas } \\
\text { participam NG2 } \\
\text { D=0 - empresas não } \\
\text { participam do NG2 }\end{array}$ \\
\hline $\begin{array}{l}\text { Tamanho (TAM) } \\
\text { (Independente) }\end{array}$ & Tamanho & Ativo Total & Logaritmo de Ativo Total \\
\hline $\begin{array}{l}\text { Alto Impacto (AI) } \\
\text { (Independente) }\end{array}$ & Adesão ao Al & $\begin{array}{l}\text { Empresas que } \\
\text { participam do Al }\end{array}$ & $\begin{array}{c}\text { Variável dummy } \\
\mathrm{D}=1 \text { - empresas } \\
\text { participam } \mathrm{Al} \\
\mathrm{D}=0 \text { - empresas não } \\
\text { participam do Al }\end{array}$ \\
\hline $\begin{array}{c}\text { Relatório de } \\
\text { sustentabilidade } \\
\text { (RS) } \\
\text { (Independente) }\end{array}$ & Divulgam RS & $\begin{array}{c}\text { Empresas que } \\
\text { divulgam RS }\end{array}$ & $\begin{array}{c}\text { Variável dummy } \\
\mathrm{D}=1 \text { - empresas que } \\
\text { divulgam } \mathrm{RS} \\
\mathrm{D}=0 \text { - empresas que não } \\
\text { divulgam } \mathrm{RS}\end{array}$ \\
\hline $\begin{array}{c}\text { ADCR } \\
\text { (Independente) }\end{array}$ & Accrualsdiscricionário & $\begin{array}{l}\text { Acumulações } \\
\text { discricionárias }\end{array}$ & Resíduos das regressões \\
\hline
\end{tabular}

Fonte: Adaptado de Nunes, Teixeira e Nossa (2009).

Assim, três regressões logísticas foram utilizadas para avaliar a relação entre a participação no ISE e QIC.

Na primeira regressão, foram utilizados os accruals discricionários como variável dependente (proxy de qualidade) e como variável 
independente, e a participação no ISE (proxy de sinalização de sustentabilidade) sem a inclusão das variáveis de controle, exceto pelas dummies dos setores.

$$
\mathrm{ADCR}_{\mathrm{it}}=\alpha+\beta_{0} \mathrm{ISE}_{\mathrm{it}}+\sum_{n=1}^{18} \beta \mathrm{nSETOR} \text { it }+\varepsilon_{\mathrm{it}}
$$

Na segunda regressão, foi incluída a ADCR (proxy de qualidade) como variável dependente. Já como variáveis independentes, foram utilizados o Relatório de Sustentabilidade (RS), Alto Impacto Ambiental (AI), Níveis de Governança 1 e 2 (NG1) (NG2), Novo Mercado (NM) e Tamanho (TAM), sem a inclusão do ISE, para fins de análise, interpretação e verificação de possíveis diferenças no modelo.

$$
\begin{aligned}
& A D C R_{i t}=\alpha+\beta_{0} T_{A M}+\beta_{1} R S_{i t}+\beta_{2} N G 1_{i t}+\beta_{3} N G 2_{i t}+\beta_{4} N_{i t}+\beta_{5} A I_{i t}+ \\
& \sum_{n=1}^{18} \beta n \text { SETOR }_{i t}+\varepsilon_{i t}
\end{aligned}
$$

Para a terceira regressão, foram utilizados os mesmos parâmetros da segunda equação, com a inclusão da participação no ISE.

$$
\begin{aligned}
& \mathrm{ADCR}_{\mathrm{it}}=\alpha+\beta_{0} \mathrm{ISE}_{\mathrm{it}}+\beta_{1} \mathrm{TAM}_{\mathrm{it}}+\beta_{2} \mathrm{RS}_{\mathrm{it}}+\beta_{3} \mathrm{NG} 1_{\mathrm{it}}+\beta_{4} \mathrm{NG} 2_{\mathrm{it}}+ \\
& \beta_{5} \mathrm{NM}_{\mathrm{it}}+\beta_{6} \mathrm{Al}_{\mathrm{it}}+\sum_{\mathrm{n}=1}^{18} \beta \mathrm{nSETOR} \text { it }+\varepsilon_{\mathrm{it}}
\end{aligned}
$$

\section{Análise dos resultados}

Para interpretar os dados, faz-se necessária uma análise empírica como forma de fomentar os resultados encontrados sob égide da investigação da QIC, apreciando os relatórios de sustentabilidade colhidos na BM\&FBovespa, visando elucidar a questão de pesquisa formulada.

$\mathrm{Na}$ tabela 1 utilizaram-se os accruals discricionários como proxy da QIC (variável dependente) e como proxy de sinalização de sustentabilidade o ISE (variável independente) sem a inclusão das variáveis de controle, controlando apenas por setores.

$$
\mathrm{ADCR}_{\mathrm{it}}=\alpha+\beta_{0} \mathrm{ISE}_{\mathrm{it}}+\sum_{\mathrm{n}=1}^{18} \beta \mathrm{SETOR} \text { it }+\varepsilon_{\mathrm{it}}
$$


TABELA 1: Variável dependente accruals discricionários e variável independente ISE.

\begin{tabular}{l|c|c|c}
\hline Variável & Coeficiente & Estatística de Testes $(\mathrm{t})$ & $\mathrm{P}$-value \\
\hline ISE & $-0,0406$ & $-2,71$ & $0.00^{* * *}$ \\
\hline Constante & 0.147 & 8.80 & $0.00^{* * *}$ \\
\hline Dummy Setor & $\mathrm{SIM}$ & Estatística-F & 3.29 \\
\hline$N^{\circ}$ Observações & 797 & $\mathrm{R}^{2}$ & 0.07 \\
\hline
\end{tabular}

$\mathrm{ADCR}=$ accruals discricionário no ano da empresa; ISE = 1 para a empresaparticipante da carteira e 0 quando não participa no período, e= controlando pelos de setores de 1 a 18 . Fonte: elaborado pelo Autor.

***1\% significância

Os resultados da tabela 1 apresentaram-se estatisticamente significantes, pois indica que empresas sustentáveis pertencentes à carteira ISE apresentam menor nível de gerenciamento de resultados, se comparadas às demais companhias abertas.

Desta forma, é possível confirmar estatisticamente que uma diminuição dos accruals discricionários gera um aumento na QIC, demonstrando a existência da relação entre as variáveis e o ISE, pois, para uma empresa participar desta carteira, também são levadas em consideração as mesmas dimensões, como tamanho, níveis de governança, divulgação de relatórios de sustentabilidade, alto impacto ambiental, e algumas destas dimensões são determinantes da qualidade da informação contábil.

Assim, ao relacionar as principais dimensões (critérios) para ingresso no ISE e o gerenciamento de resultados, foi identificado que estas variáveis de fato possuem relação com a qualidade da informação contábil.

Na tabela 2 foi utilizado o gerenciamento de resultados (GR) como variável dependente, ou seja, proxy de qualidade. Para explicá-la, utilizaram-se como variáveis independentes as dimensões consideradas para entrada no ISE, como o Relatório de Sustentabilidade (RS), Tamanho (TAM), os níveis 1 e 2 dos atributos de governança corporativa (NG1) e (NG2), Novo Mercado (NM) e Alto Impacto (Al), que funcionarão como mecanismos de controle, sem a inclusão do ISE. 


$$
\begin{gathered}
A D C R_{i t}=\alpha+\beta_{0} \text { TAM }_{i t}+\beta_{1} R_{i t}+\beta_{2} N G 1_{i t}+\beta_{3} N G 2_{i t}+\beta_{4} N_{i t}+\beta_{5} A_{i t}+ \\
\sum \beta n_{n=1}^{18} \beta n S E T O R_{i t}+\varepsilon_{i t}
\end{gathered}
$$

TABELA 2: Variável dependente: accruals discricionários e variáveis independentes TAM, RS, NG1, NG2, NM e Al sem a inclusão do ISE.

\begin{tabular}{|c|c|c|c|}
\hline Variáveis & Coeficiente & Estatística de Testes $(\mathrm{t})$ & $\mathrm{P}$ - value \\
\hline TAM & $-0,012$ & $-4,080$ & $0.00^{* * *}$ \\
\hline RS & $-0,214$ & $-1,920$ & $0,05^{* *}$ \\
\hline NG1 & $-0,003$ & $-0,200$ & 0.84 \\
\hline NG2 & $-0,049$ & $-2,430$ & $0.01^{* * *}$ \\
\hline NM & $-0,017$ & $-1,670$ & $0,09^{*}$ \\
\hline Al & 0,020 & $-0,054$ & 0.58 \\
\hline Constante & 0.324 & 7.86 & $0.00^{* * *}$ \\
\hline Dummy Setor & SIM & Estatística-F & 4,84 \\
\hline$N^{\circ}$ Observação & 797 & $R^{2}$ & 0,13 \\
\hline
\end{tabular}

$\mathrm{ADCR}=$ accruals discricionários no período $\mathrm{t}$ da empresa $\mathrm{i}$, TAM = Ativo Total da empresano período , RS = variável dummy 1 quando a empresa no período divulga o relatório e 0 quando não divulga NG1 = variável Dummy 1 se a empresa i participante do nível 1 e 0 quando não participante no período, NG2 = variável dummy 1 a empresa i participante do nível 2 e 0 quando não participante no período t $\mathrm{NM}=$ variável dummy 1 empresa participante e 0 quando não participante, $\mathrm{Al}=$ variável dummy 1 para atividade empresarial no período de alto impacto ambiental e 0 quando não ser de alto impacto ambiental, = Controlando pelo número de setores de 1 a 18 .

Fonte: Elaborado pelo Autor

${ }^{* * *},{ }^{* *},{ }^{*}$ estatisticamente significante aos níveis de $1 \%, 5 \%$ e $10 \%$.

Os resultados da tabela 2 mostraram que as variáveis TAM, RS, NG2 e NM possuem significância na estimação, corroborando com os artigos de Gamerschlang, Moller e Verbeeten (2011), que encontraram relação estatística entre o Gerenciamento de Resultados (GR) e a variável tamanho, sugerindo que empresas maiores possuem maior organização em operacionalização e, portanto, produziriam uma melhor qualidade da informação e menor gerenciamento de resultados.

Os dados da tabela 2 corroboram com a pesquisa de Dechow, Ge e Schrand (2010) e Byard e Weintrop (2006) ao adicionar a variável 
sustentabilidade como proxie de qualidade da informação contábil, assim como as variáveis tamanho da empresa e os atributos de governança corporativa.

Os resultados sinalizam que as empresas de maior porte possuem um menor gerenciamento de resultados. Já as demais variáveis independentes NG2 foram significantes ao nível de 5\%, e as variáveis RS e NM mostraram-se significantes ao nível de 10\%, contudo, NG1 e Al não mostraram qualquer correlação estatística.

A pesquisa também contribui com os achados de Pereira, Carvalho e Parente (2011) em que as empresas mais rentáveis são as que possuem um melhor desempenho econômico e um ambiente favorável, pois acabam por evidenciar mais informações ambientais no que tange aos indicadores econômicos, tornando-se empresas rentáveis e competitivas, capazes de se manterem no mercado.

$\mathrm{Na}$ tabela 3 foram utilizados os parâmetros da tabela 2, com a inclusão apenas do ISE no modelo da regressão como variável independente, para fins de verificar possíveis diferenças no modelo.

$$
\begin{aligned}
& \mathrm{ADCR}_{\mathrm{it}}=\alpha+\beta_{0} \mathrm{ISE}_{\mathrm{it}}+\beta_{1} \mathrm{TAM}_{\mathrm{it}}+\beta_{2} \mathrm{RS}_{\mathrm{it}}+\beta_{3} \mathrm{NG} 1_{\mathrm{it}}+\beta_{4} \mathrm{NG}_{\mathrm{it}}+\beta_{5} \\
& \mathrm{NM}_{\mathrm{it}}+\beta_{6} \mathrm{Al}_{\mathrm{it}}+\sum_{\mathrm{n}=1}^{18} \quad \beta n \mathrm{SETOR} \\
& \mathrm{it}
\end{aligned}
$$

TABELA 3: Variável dependente: accruals discricionários variáveis

\begin{tabular}{|c|c|c|c|}
\hline Variáveis & Coeficiente & Estatística de Testes (t) & $\mathbf{P}$-value \\
\hline ISE & 0,0015 & 0,09 & $-0,92$ \\
\hline TAM & $-0,1226$ & $-4,05$ & $-0,00^{* * *}$ \\
\hline RS & $-0,0122$ & $-1,86$ & $-0,06^{*}$ \\
\hline NG1 & $-0,0035$ & $-0,20$ & 0,83 \\
\hline NG2 & $-0,0498$ & -242 & $0,01^{* * *}$ \\
\hline NM & $-0,0179$ & $-1,67$ & $0,09^{*}$ \\
\hline $\mathrm{Al}$ & $-0,0195$ & $-0,54$ & 0,58 \\
\hline Constante & 0.325 & 7.77 & $0.00^{* * *}$ \\
\hline Dummy Setor & SIM & Estatística-F & 4,65 \\
\hline No Observações & 797 & $R^{2}$ & 0,13 \\
\hline
\end{tabular}
independentes, ISE, TAM, RS, NG1, NG2, NM e AI.

Fonte: Elaborado pelo Autor

***, * estatisticamente significante aos níveis de $1 \%$ e $10 \%$ 
Osresultados daregressãoapresentadosnatabela3 3 permaneceram estatisticamente relacionados com a variável independente accruals aos níveis de $1 \%$ e $10 \%$ respectivamente, já a variável independente ISE inserida no modelo não foi estatisticamente correlacionadas.

Desse modo, levantam-se evidências de que não é a entrada no ISE que faz com que as empresas gerenciam menos os resultados, mas sim os critérios relacionados no questionário respondido pelas empresas de acesso ao ISE, como Tamanho, Relatório de Sustentabilidade, Nível de Governança 2 e Novo Mercado.

Assim, estes critérios que levam empresas a participarem do ISE por estarem relacionados com o menor gerenciamento de resultados, demonstram que não é o ISE que gera menor GR, mas sim os critérios de escolhas. Portanto, o ISE é quase uma proxy indireta de características de empresas com maior qualidade da informação contábil.

O ISE considera várias dimensões como: tamanho, níveis de governança, divulgação de relatório de sustentabilidade, empresas de alto impacto ambiental e algumas destas dimensões são determinantes da qualidade da informação contábil.

Assim, ao relacionar as principais dimensões (critérios) para ingresso no ISE e o gerenciamento de resultados (GR), identificaram-se quais dimensões são as que, de fato, possuem relação com a qualidade da informação contábil.

Tanto é que na tabela 3, ao acrescentar o ISE ao modelo junto a estas regressões, o indicador deixa de apresentar significância estatística dado que as outras variáveis independentes já explicam a relação com a QIC.

Desse modo, é em função desses critérios que uma empresa pode participar do ISE, como empresas que divulgam relatório de sustentabilidade, estejam participantes dos atributos de governança, alto impacto e tamanho, porém, alguns destes critérios estão também ligados ao gerenciamento de resultados, como tamanho, divulgação de relatórios de sustentabilidade e atributos de governança. 
Estes resultados corroboram com os achados de Correia, Amaral e Louvet (2011), em que as empresas pertencentes à governança usam a qualidade da informação, como meio de diminuir a assimetria de informações entre as partes envolvidas, melhorando, por conseguinte os possíveis conflitos de interesse.

Outro achado da pesquisa contribui com os resultados dos artigos de Dechow, Ge Schrand (2010) e Byard e Weintrop (2006), uma vez que identificam que as empresas que divulgam o relatório de sustentabilidade possuem menor gerenciamento de resultados e, por conseguinte, maior qualidade da informação contábil.

Isso pode ocorrer pela potencial relação entre a divulgação do relatório de sustentabilidade como uma forma de transparência de negócio, demonstrando que as empresas envolvidas com a sustentabilidade possuem maior facilidade de diferenciarem das demais empresas e aumentando, por conseguinte, a confiança do público.

Os resultados evidenciam uma relação positiva entre o ISE e a qualidade da informação contábil, indicando serem essas empresas mais avaliadas pelo mercado, sendo a qualidade um fator a ser considerado pelos investidores ao escolher as ações.

O ISE, portanto, representa para os investidores ganhos em termos de resumo e redução do custo de transação, podendo ser visto pelo mercado como uma métrica de qualidade da informação contábil (QIC).

\section{Considerações finais}

O estudo testou se as empresas que estavam inseridas no ISE no período de 2010 a 2012 são as que possuem melhor QIC em relação aos demais setores da BM\&FBovespa.

Os achados da pesquisa sugerem uma relação negativa entre os accruals discricionários e a participação no ISE, ou seja, as empresas 
classificadas como "sustentáveis" apresentam maior Qualidade da Informação Contábil.

Porém, o ISE considera várias dimensões, como tamanho, níveis de governança, divulgação de relatório de sustentabilidade e alto impacto ambiental, e algumas destas dimensões também são determinantes da qualidade da informação contábil.

Assim, ao relacionar as principais dimensões (critérios) para ingresso no ISE e o gerenciamento de resultados, foram identificadas as dimensões que, de fato, possuem relação com a qualidade da informação contábil, quais sejam: tamanho (TAM) das empresas, empresas que divulgam relatório de sustentabilidade (RS), empresas do Novo Mercado (NM) e as do nível de Governança Corporativa (NG2).

Os resultados da pesquisa indicam que as empresas inclusas no ISE possuem menor nível de Gerenciamento de Resultados, portanto, sinalizam para o mercado maior Qualidade da Informação Contábil em relação às empresas não participantes da carteira.

Desse modo, pode-se afirmar que não seria a entrada no ISE que levaria as empresas a gerenciar menos os resultados, mas sim os critérios relacionados no questionário respondido pelas empresas de acesso ao ISE. O ISE, portanto, seria uma proxy indireta de características de empresas com maior QIC.

A identificação de que as empresas que divulgam o relatório de sustentabilidade possuem menor nível de gerenciamento de resultados, e, por conseguinte, maior qualidade da informação contábil, corrobora com as pesquisas nacionais e internacionais ao adicionar a variável sustentabilidade como uma das proxies de qualidade da informação contábil, assim como as variáveis tamanho da empresa e atributos de governança corporativa.

Dessa forma, a participação no ISE pode ser interpretada como uma métrica de sinalização para capturar a qualidade da informação, possibilitando ganhos aos usuários em termos de redução de custos de transação para os diversos grupos de stakeholders. 
Ressalta-se que os resultados da pesquisa não permitem conclusões definitivas, pois existem algumas limitações, como: (a) a estimação dos accruals discricionários e (b) o período da amostra.

Novas pesquisas poderão comparar a carteira ISE, composta por 40 (quarenta) empresas, em relação às 160 empresas remanescentes das 200 (duzentas) ações mais negociadas na BM\&FBovespa.

\section{Referências}

ADACHI, A. L.; SANTOS, A. F. UREL, K. M.; BIAGI, L. H. M.; VENDRAME, F. C.. A TI nas empresas. Lins - SP: Unisalesiano., 2009. Disponível em: <http://www.unisalesiano.edu.br/encontro2009/ trabalho/aceitos/CC30069895899.pdf>, Acesso em: 26 fev. 2014.

ANDRADE, L. P.; BRESSAN, A. A.; IQUIAPAZA, R. A.; MOREIRA, B. C. M. Determinantes de adesão ao índice de sustentabilidade empresarial da BM\&FBovespa e sua relação com o valor da empresa. Revista Brasileira Finanças, São Paulo. v. 11, n. 2, p. 181-213, abr/ jun. 2013.

ANTUNES, G. A.; COSTA, F. M. Governança e qualidade da informação contábil: uma investigação utilizando empresas bras. que aderiram ou não aos níveis diferentes. de governança da BOVESPA. In: ENCONTRO DAANPAD, 31, 2007, Rio de Janeiro, RJ. Anais... Rio de Janeiro: ANPAD, set. 2007.p. 22-26,

ANTUNES, G. A.; TEIXEIRA, A. J. C; COSTA, F. M; NOSSA, V. Efeitos da adesão aos níveis de governança da BOVESPA na qualidade da informação contábil. ASAA Advances in Scientific and Applied Accounting, São Paulo. v. 3, n.1, p.109-138, 2010.

BACHMANN, R. K. B.; CARNEIRO, L. M.; ESPEJO, M. M. S. B. Evidenciação de informações ambientais: proposta de um indicador a partir da percepção de especialistas. Revista Contabilidade Organizações, São Paulo. v. 7, n. 17, p. 36-47, 2013. 
BIDDLE, G. C.; HILARY, G. Accounting quality and firm-level capital investment. Accounting Review.Hong Kong, v.81, n. 5, p. 963-982. Oct. 2006.

BYARD, D.; LI, Ying; WEINTROP, J. Corporate governance and the quality of financial analysts information. Journal of Accounting and Public Policy. New York. v. 25, n. 5, p. 609-625, Sep. 2006.

BOUBAKER, S.; SAMI, H.. Multiple large shareholders and earnings informativeness. Review of Accounting and Finance. França, v.10, n. 11, p. 246-266, 2011.

BM\&FBOVESPA. Mercado: ações - índices. 2010. Disponível em: <http://bovespa.com.br>. Acesso em:10 dez. 2013.

BM\&FBOVESPA. Novo valor: sustentabilidade nas empresas - Como começar, quem envolver e o que priorizar. Disponível em: <http://www. bmfbovespa.com.br/empresas/download/guia-de-sustentabilidade. pdf>. Acesso em: $11 \mathrm{dez} .2013$.

\section{BM\&FBOVESPA. Indice de sustentabilidade empresarial:} metodologia completa. Disponível em: <http://www.bmfbovespa.com. br/Indices/download/ISE.pdf>. Acesso em: 27 dez. 2013.

BM\&FBOVESPA. Índice de sustentabilidade empresarial. Disponível em: <www.bmfbovespa.com.br/indices/download/metodologia-ise.pdf>. Acessoem 27 de dez. 2013.

COLARES, A. C. V.; BRESSAN, V. G. F.; LAMOUNIER, W. M.; BORGES, D. L.O balanço social como indicativo socioambiental das empresas do índice de sustentabilidade empresarial da BM\&FBovespa. Revista de Contabilidade do Mestrado em Ciências Contábeis da UERJ, Rio de Janeiro, v. 17, n. 1, Ed. Especial, p. 83100, out, 2012.

CORREIA, L. F; AMARAL, H. F; LOUVET, P. Um índice de avaliação da qualidade da governança corporativa no Brasil. Revista Contabilidade e Finanças, São Paulo, v. 22, n. 55, p. 45-63, jan./fev./ mar./abr. 2011. 
DECHOW, P. M; SLOAN, R.; SWEENY, A. Causes and consequences of earnings manipulation: an analysis of firms subject to enforcement actions by the SEC. Contemporary Accounting Research, [S.I], Arizona, v. 13, n. 1, p. 1-36, Spring 1996.

DECHOW, P. M; SLOAN, R., SWEENY, A. Detecting earnings management. The Accounting Review. Arizona, v. 70, n. 02, p.193225, Apr.1995.

DECHOW, P. M; GE, W.; SCHRAND, C. Understanding earnings quality: a review of proxies, their determinants and their consequences. Journal of Accounting and Economics. Arizona, v. 50, n. 2-3. p. 344401, Dec. 2010.

ELKINGTON, J. Enter the Triple Bottom Line. In: HENRIQUES, A.; RICHARDSON, J. The triple bottom line, does it all add up? Assessing the sustainability of Bbsiness and CSR. London: Earthscan Publications Ltd, 2004. p. 1-16.

FERNANDES, S.M. A influência do disclosure ambiental voluntário no custo da dívida. Revista de administração e Inovação, São Paulo, v. 10, n.3, p. 165-183, jul./set. 2013.

GALLON, A. V.; ENSSLIN, S. R. Evidenciação estratégica dos pilares da sustentabilidade empresarial: investigação no relatório da administração das empresas que compõem o ISE. Revista Pensar Contábil. Rio de Janeiro, v.10, n. 41, p. 33-45.jun./jul, 2008.

GARCIA, A. S.; ORSATO, R. J. Índice de sustentabilidade empresarial: porque participar? In: ENCONTRO DA ANPAD, 37., 2013, Rio de Janeiro, RJ. Anais... Rio de Janeiro: ANPAD, 2013. p. 7-11.

GOMES, P. H. V.; LUCA, M. M. M.; VASCONCELOS, A. C.; PONTE, V. M. R. R. Fatores determinantes do disclosure voluntário sob o enfoque da sustentabilidade: uma análise das empresas dos países do BRIC. Revista de Gestão Social e Ambiental -RGSA, São Paulo, v.9, n.2, p.70-87, maio/ago.,2015.

JONES, J., Earnings management during import relief investigations. 
Journal of Accounting Research. Chicago - EUA. v. 2, n 2, p. 193228, Autumm,1991.

CENTRO DE ESTUDOS EM SUSTENTABILIDADE da FGV/EAESP (Gvces). Pesos dos critérios ISE 2012/2013. Disponível em: <http:// gvces.com.br/pesos-dos-criterios-ise-2012-2013?locale=pt-br>. Acesso em: 25. jan. 2014.

MACHADO, M. R.; MACHADO, M. A. V.; CORRAR, L. J. Desempenho do Índice de Sustentabilidade Empresarial (ISE) da Bolsa de Valores de São Paulo. Revista Universo Contábil, FURB, Blumenal, v. 5, n. 2, p. 24-38, abr./jun., 2009.

MARCONDES, A. W.; BACARJI, C. D. ISE - Sustentabilidade no Mercado de Capitais. São Paulo: Report Ed., 2010. p. 178. Disponível em: <http://bibliotecadigital.fgv.br/dspace/handle/10438/15424>. Acesso em: 20 abr. 2014.

MELO, R. A.; MANHÃES, J. V. P.; SILVA; MACEDO, M. A..S.

Desempenho do Índice de Sustentabilidade Empresarial (ISE) da Bolsa de Valores de São Paulo sob a ótica do Índice de Sharpe. Revista de Economia \& Gestão, Belo Horizonte. v. 12, n. 28, p. 75-104, jan./abr. 2012.

MOON, S.; DE LEON, P. Contexts and Corporate Voluntary Environmental behaviors. Organization \& Enviroment, Colorado EUA, v. 20, n. 4, p. 480-496, Dec. 2007.

MOTA, M. O.; MAZZA, A. C. A.; OLIVEIRA, F. C. Uma análise dos relatórios de sustentabilidade no âmbito ambiental do Brasil: sustentabilidade ou Camuflagem? Revista de Administração e Contabilidade da Unisinos - BASE, Rio Grande do Sul, v. 10, n. 1, p. 69-80, jan./mar, 2013.

NASCIMENTO, A. M.; REGINATO, L.. Divulgação da informação contábil, governança corporativa e controle organizacional: Uma relação necessária, Revista Universo Contábil, Blumenau, v. 4, n. 3, p. 25-47, jul./set. 2008 . 
NOBRE, F. S.; RIBEIRO, R. E. M. Cognição e sustentabilidade: estudo de casos múltiplos no índice de sustentabilidade empresarial da BM\&FBOVESPA, RAC. Rio de Janeiro, v. 17, n. 4, art. 6, p. 499-517, jul./ago. 2013.

NOVELINI, C. P; FREGONESI, M. S. F. A. Análise da divulgação de inform. sobre investimentos sociais por empresas que se declaram socialmente responsáveis. Revista de Contabilidade e Organizações, São Paulo, v. 7, n. 17, p. 89-101, 2013.

NUNES, J. G; TEIXEIRA, A. J. C. NOSSA, V.; GALDI, F. C. Análise das variáveis que influenciam a adesão das empresas ao Índice Bovespa de Sustentabilidade Empresarial. Revista de Administração e Contabilidade da Unisino. São Paulo, v. 7, n. 4, p. 328-340, 2010. NUNES, T. C. S.; NOVA, S. C.; CORNACCHIONE, E.; GARCIA, S. Are sustainable companies less risky and more profitable? Revista de Administração - RAUSP, São Paulo, v. 47, n.3, p. 422-435, jul./ago/ set. 2012.

PAULO, E.; CORRAR, L. J.; MARTINS, E. Detectando gerenciamento de resultados pela análise de diferimento tributário. RAE, São Paulo, v. 47, n. 1, p. 46-59, jan/mar. 2007.

PAULO,I. I. S. L. M.; CAVALCANTE, P. R. N.; PAULO, E. Relação entre qualidade da Auditoria e Conservadorismo Contábil nas empresas brasileiras, Revista de Educação e Pesquisa em Contabilidade - REPEC. São Paulo, v.7, n. 3, art. 6, p. 306-327, jul/ set, 2013.

PEREIRA, G.; CARVALHO, F. N.; PARENTE, G. V.. Desempenho econômico e evidenciação ambiental: análise das empresas que receberam o Prêmio Rumo à Credibilidade 2010, Revista Catarinense da Ciência Contábil,- CRCS. Santa Catarina, v.10, n. 30, p. 9-26, ago./nov. 2011.

REZENDE, I. A. C.; NUNES, J. G. PORTELA, S. S.; Um estudo sobre o desempenho financeiro do índice BOVESPA de Sustentabilidade 
Empresarial. Revista de Educação e Pesquisa em Contabilidade REPEC. Brasília, DF, v. 2, n. 1, art. 4, p. 71-93. jan./abr. 2008.

RIBEIRO, M. R. F.; TRETER, J. Governança Corporativa e a relevância da informação contábil um estudo de caso em uma cooperativa.Disponível em: <http://www.unicruz.edu.br/site/cursos/ contabeis/artigos/Artigos\%202011/Mara\%20Rubia\%20Fagundes.pdf>. Acesso em: 27. jan. 2014.

SANTOS, P. S. A.; DANI, A. C; RIBEIRO FILHO, J. T.Qualidade Informacional Contábil das Companhias de Tecnologia da Informação Listadas na BM\&FBovespa. R. Gestão \&Tecnologia, Pedro Leopoldo, v. 11, n. 2, p. 1-18, jul./dez. 2011.

SANTOS, L. P. G.; LIMA, G. A. S. F.; FREITAS, S. C.; LIMA, I. S.Efeito da Lei 11.638/07 sobre o conservadorismo condicional das empresas listadas BM\&FBOVESPA. Revista de Contabilidade e Finanças. São Paulo, v. 22, n. 56, p. 174-188, maio/jun./jul./ago. 2011.

SILVA, G. R; COSTA, F. M. Qualidade da Informação Contábil e Sustentabilidade: um Estudo nas Companhias Brasileiras Listadas na BM\&FBovespa. In: ENCONTRO DAANPAD - ENANPAD, 39., set. 2015, Belo Horizonte, MG. Anais...Belo Horizonte: ANPAD, 2015.p. 2-17.

SPENCE, M. Job Market Signalling, Quarterly Journal of Economics, Oxford University. v. 87, n. 1, p. 355-374, Fev. 1973.

SZÜSTER, N.; SZÜSTER, F. R.; SZÜSTER, F. R. Contabilidade: atuais desafios e alternativa para seu melhor desempenho. Revista de Contabilidade \& Finanças - USP, São Paulo, v. 16, n, 38, p. 20-30. maio/ago. 2005.

TEIXEIRA, E.A.; NOSSA, V.; FUNCHAL, B.. O índice de sustentabilidade empresarial (ISE) e os impactos no endividamento e na percepção de risco. Revista Contabilidade \& Finanças, São Paulo. v. 22, n. 55, p. 29-44. jan./abr. 2011. 
TERUEL, P. J. G.; SOLANO, P. M.; BALLESTA, J. P. Accruals quality and corporate cash holdings. Accounting and Finance. Espanha. v. 49, n. 1, p. 95-115, Mar. 2009.

WARFIELD, T., J.; WILD, K. Managerial Ownership, Accounting

Choices and Informativeness of Earnings. Journal of Accounting and Economics. Londres. UK. v 20. n. 1, p. 61-91. Jul. 1995.

Data de Submissão: 22/12/15

Data de Aprovação: 07/09/16 